\title{
Designing hybrid flexure systems and elements using Freedom and Constraint Topologies
}

\author{
J. B. Hopkins \\ Lawrence Livermore National Laboratory, 7000 East Avenue L-223, Livermore, CA 94551, USA \\ Correspondence to: J. B. Hopkins (jonathanbhopkins@gmail.com)
}

Received: 20 March 2013 - Accepted: 12 June 2013 - Published: 1 October 2013

\begin{abstract}
In this paper we introduce the principles necessary to synthesize hybrid flexure systems and elements. Flexure systems consist of rigid bodies that are joined together by flexure elements that elastically deform to guide the system's rigid bodies with desired degrees of freedom (DOFs). The principles introduced here for synthesizing hybrid flexure systems and elements are extensions of the Freedom and Constraint Topologies (FACT) synthesis approach. FACT utilizes a comprehensive library of geometric shapes from which designers can rapidly consider and compare a multiplicity of flexure concepts that achieve any desired set of DOFs. Prior to this paper, designers primarily used these shapes to synthesize parallel and serial flexure systems and elements. With this paper, designers may now use these same shapes to synthesize more general flexures that consist of various combinations of parallel and serial systems and elements (i.e., hybrid configurations). As such, designers can access a larger body of flexure solutions that satisfy demanding design requirements. Instructions for helping designers utilize or avoid the advantages and challenges of over-, under-, and exactconstraint are also provided. Hybrid systems and elements are analysed and designed as case studies.
\end{abstract}

\section{Introduction}

Flexure systems consist of rigid bodies that are joined together by flexure elements (Smith, 2000). These elements are directionally compliant and thus guide the system's rigid bodies to move in prescribed directions called degrees of freedom (DOFs) via elastic deformation. There are three kinds of flexure systems - parallel, serial, and hybrid (Fig. 1). Parallel systems consist of a single rigid body that is directly connected to a fixed or grounded body by flexure elements. Serial systems consist of two or more parallel systems sequentially stacked in a chain-like configuration. Hybrid systems consist of various combinations of parallel and serial systems joined together. Schematic representations of general parallel, serial, and hybrid systems are shown in the top portion of Fig. 1. Rigid bodies are shown as rectangles and flexure elements are shown as springs. Fixed or grounded bodies are labelled $G$, intermediate bodies are labelled $I$, and motion stages are labelled $S$. Example parallel, serial, and hybrid flexure systems are shown in the bottom portion of the same figure. Note that the flexure elements of these examples are blade flexures and that the hybrid example consists of two serial flexure systems arranged in parallel.

Similar to flexure systems, flexure elements may be categorized as parallel, serial, or hybrid. Examples of common parallel flexure elements are wire, blade, living hinge, and notch flexures. These elements are shown as $E_{1}$ through $E_{4}$ in Fig. 2. Parallel flexure elements impose constraining forces directly through all parts of their geometry to the rigid bodies that they join. As such, if constraining forces are represented by blue lines (called constraint lines in this paper) with an axis that is collinear with the force's line of action, the entire compliant portion of a parallel element's geometry can be filled with these lines passing directly from one of its rigid bodies to the next. Consider element $E_{1}$ in Fig. 2 for example. Only one constraint line fits within its geometry because a wire flexure is only capable of imposing an appreciable constraining force along its axis. The blade flexure $E_{2}$, on the other hand, can impose many forces on its rigid bodies. These forces act along the axes of constraint lines that span between these bodies and pass through the planar geometry of the blade (only a few such constraint line examples are 


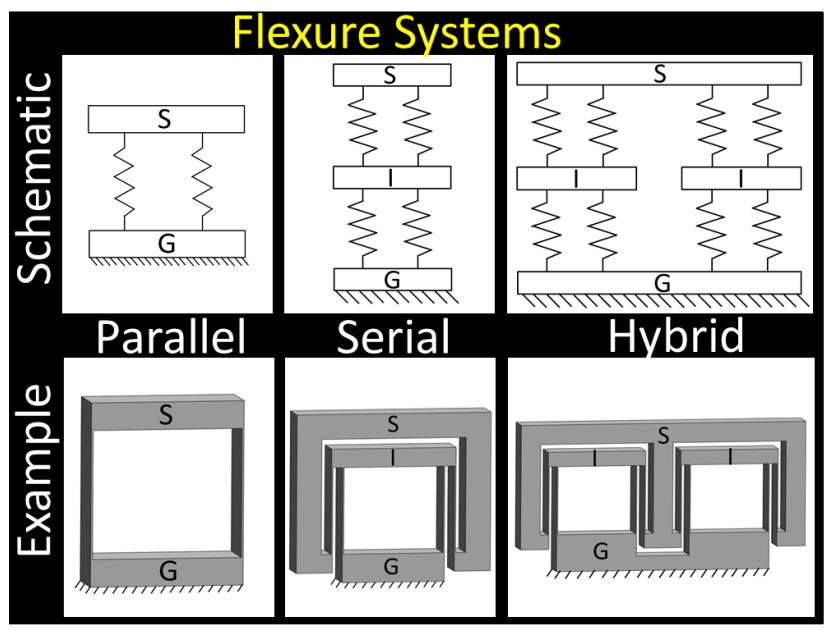

Figure 1. Three kinds of flexure systems - parallel, serial, and hybrid.
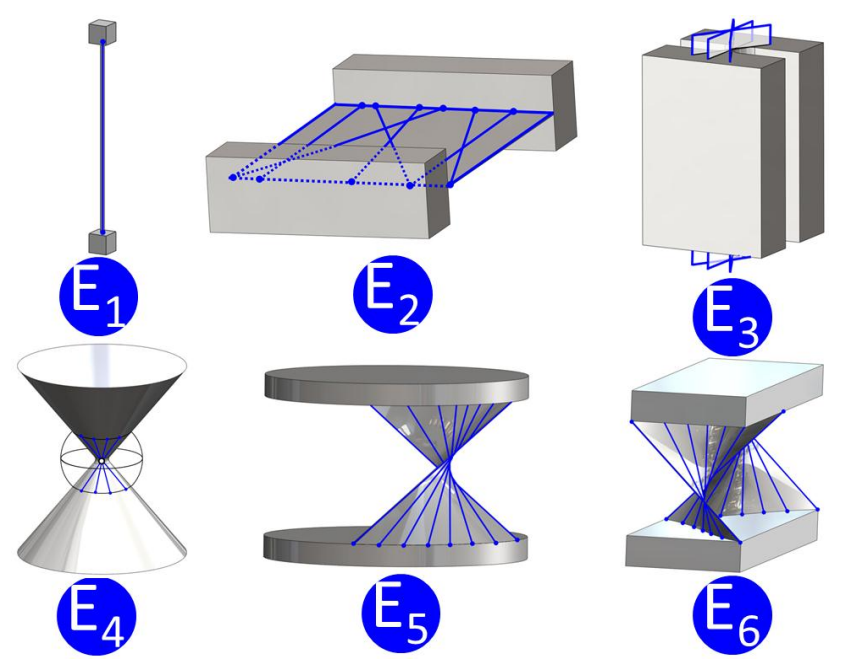

Figure 2. Parallel flexure element examples.

shown in the figure). Likewise, the living hinge and notch flexures, $E_{3}$ and $E_{4}$, from Fig. 2 may impose forces on their rigid bodies that act along the axes of constraint lines that span between these bodies and pass entirely through the compliant portion of their geometries. For $E_{3}$, these lines lie on the surfaces of intersecting planes, and for $E_{4}$, these lines lie within a sphere and intersect at a common point at the neck of the flexure. Other, less common examples of parallel flexure elements are also shown in Fig. 2. These elements, $E_{5}$ and $E_{6}$, are shown with constraint lines that fill their geometry and directly join their rigid bodies. In Figs. 1 and 3, the springs shown in the schematic portion must represent parallel flexure elements only to maintain continuity with the definitions provided here.

Serial flexure elements consist of parallel elements stacked together in serial configurations with no intermediate rigid

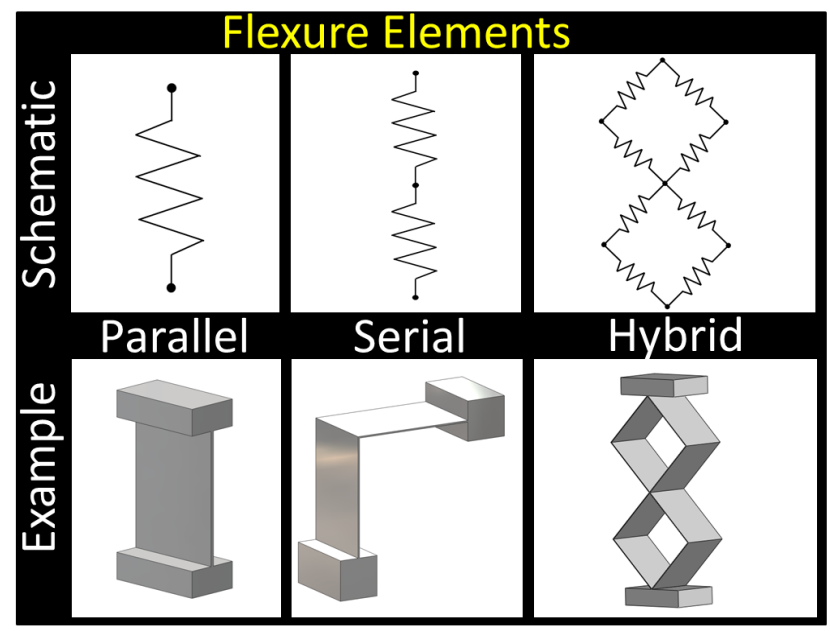

Figure 3. Three kinds of flexure elements - parallel, serial, and hybrid.

bodies in between. Thus, the junctions between these elements are depicted as black nodes instead of as rectangles in the schematic portion of Fig. 3. The example serial flexure element shown in this figure is a bent blade flexure that consists of two parallel blade flexure elements stacked in series.

Hybrid flexure elements consist of various combinations of parallel and serial elements joined together with no rigid bodies in between. The example hybrid flexure element shown in Fig. 3 consists of two pairs of serial bent blade flexure elements arranged in parallel that are stacked in series with one another. Unlike parallel flexure elements, serial and hybrid flexure elements possess geometries that cannot be entirely filled with constraint lines that directly join their rigid bodies. Note that no constraint lines can pass between the rigid bodies and remain within the geometries of either the serial or hybrid flexure element examples from Fig. 3. Many other examples of parallel, serial, and hybrid flexure elements are provided in Howell et al. (2013).

The intent of this paper is to introduce the theory necessary to synthesize hybrid flexure systems and elements that consist of various combinations of parallel and serial systems and elements of any complexity. This theory is an extension of the Freedom and Constraint Topologies (FACT) synthesis approach (Hopkins and Culpepper, 2006; Hopkins, 2007). This approach utilizes a comprehensive library of geometric shapes, similar to the shapes formed by the constraint lines within the examples of Fig. 2, which guide designers in synthesizing flexure systems and elements that achieve any desired set of DOFs. These shapes visually embody the mathematics of screw theory (Ball, 1900; Phillips, 1984, 1990) and enable designers to rapidly consider and compare a multiplicity of solution concepts in an intuitive way. The shapes of FACT have previously been applied to the design of parallel flexure systems (Hopkins and Culpepper, 2010), serial flexure systems (Hopkins and Culpepper, 2011), parallel flexure 
elements (Hopkins, 2012; Hopkins et al., 2013), and serial flexure elements (Hopkins, 2013). In this paper, we briefly review the principles of these publications and explain how these principles can be adapted and applied to the design of hybrid flexure systems and elements. Thus, this paper contains the general theory of FACT necessary to synthesize flexure-based motion stages of all configurations.

The ability to synthesize hybrid flexure systems and elements as well as those that are configured in parallel and/or serial is important because this ability allows designers to consider the entire body of flexure solution concepts that achieve a wider variety of kinematic, elastomechanic, and dynamic design requirements. With this ability, designers are guaranteed to identify multiple solutions that achieve any set of desired DOFs. The ability to synthesize hybrid flexure systems is particularly important because flexures that decouple displacement-based actuators within multi-DOF systems are often hybrid of necessity ( $\mathrm{Li}$ and $\mathrm{Xu}, 2009$; Awtar et al., 2012). Furthermore, the ability to synthesize hybrid flexure systems enables designers to create flexure-based transmission mechanisms that are capable of transforming any set of input motions into any other set of output motions with any desired transmission ratio (Hopkins and Panas, 2013). Thus, as hybrid flexure systems enable capabilities that cannot be achieved using any other configuration, the comprehensive approach provided here for synthesizing general hybrid systems significantly impacts the design of precision motion stages, nano-positioners, MEMS devices, optical mounts, and other general-purpose flexure bearings.

The intuitive approach of this paper stems from the rigorous mathematics of screw theory. The shapes of FACT are visual representations of screw systems (Ball, 1900) that consist of line geometries (Klein, 1921; Merlet, 1989) that embody a system's kinematics. Initially, screw theory was primarily used to design and analyse rigid-body spatial mechanisms and robotic manipulators (Hunt, 1978; Bothema and Roth, 1990; Murray et al., 1994; Merlet, 2000). More recently, however, screw theory has been used to design and analyse flexure systems and compliant mechanisms (Kong and Gosselin, 2004; Hao and Kong, 2013). Although the principles of FACT are similar to other significant screwtheory-based approaches for synthesizing flexure systems (Su et al., 2009; Yu et al., 2010), FACT does not require its users to be experienced with the mathematics of screw theory. The intuitive shapes of FACT are thus accessible to both novel and experienced designers in that designers are required only to visualize geometric shapes to analyse and design complex flexure systems and elements.

The specific contributions of this paper are (i) the principles of FACT are extended such that designers may analyse and design hybrid flexure systems that consist of any combination of parallel and/or serial systems, (ii) rules are provided for helping designers utilize the shapes of FACT for synthesizing hybrid systems that are either over-, under-, or exactly-constrained, (iii) the theory is provided for analysing
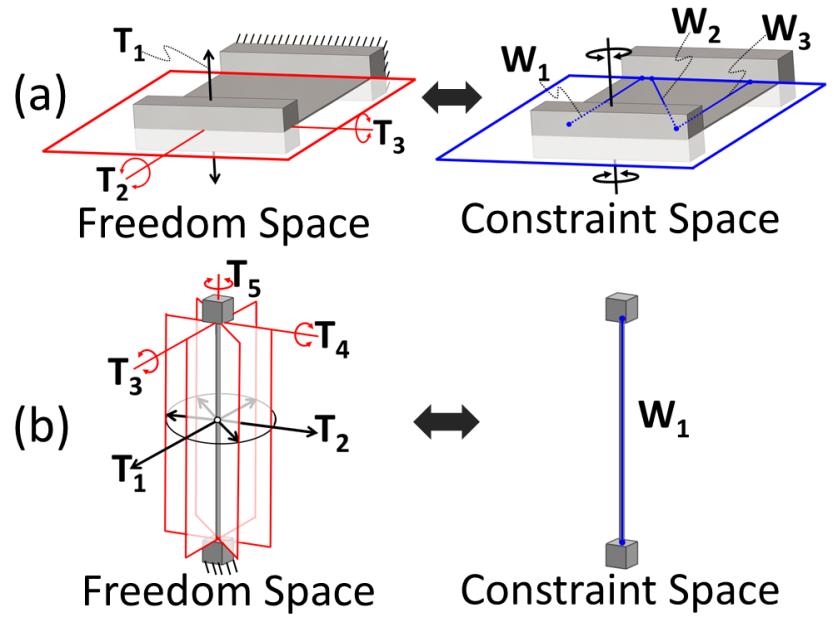

Figure 4. Freedom and constraint spaces for two flexure elements - blade and wire flexure.

and designing hybrid flexure elements that achieve the benefits of under-constrained systems without the usual disadvantages of poor dynamic characteristics, and (iv) a number of novel hybrid flexure elements are provided as examples.

\section{Fundamental principles}

This section reviews the fundamental principles of FACT necessary to analyse and synthesize parallel and serial flexure systems. Although the mathematics of these principles have been applied to rigid body mechanisms for many years, only recently have these principles been applied to the synthesis of parallel (Hopkins and Culpepper, 2006, 2010; Hopkins, 2007; Su et al., 2009; Su and Tari, 2010) and serial flexure systems (Hopkins, 2010; Hopkins and Culpepper, 2011; Su, 2011) These principles will be extended in later sections to enable designers to analyse and design hybrid flexure systems and elements using FACT.

\subsection{Freedom and constraint spaces}

Consider the blade flexure element in Fig. 4a, which possesses three DOFs - one translation and two rotations. These DOFs may be modelled using twist vectors $\boldsymbol{T}_{1}, \boldsymbol{T}_{2}$, and $\boldsymbol{T}_{3}$ respectively (Ball, 1900; Hao and McCarthy, 1998). In this paper, translations are depicted as black arrows along which bodies translate, rotations are depicted as red lines about which bodies rotate, and screws are depicted as green lines along and about which bodies simultaneously translate and rotate with a coupled pitch value. The three DOFs of the blade flexure are not the only ways its rigid body may move. It may also move with every combination of these DOFs. If these DOFs are simultaneously actuated with various magnitudes, the blade's rigid body will rotate about other lines that lie on the plane of the blade as shown highlighted red on 


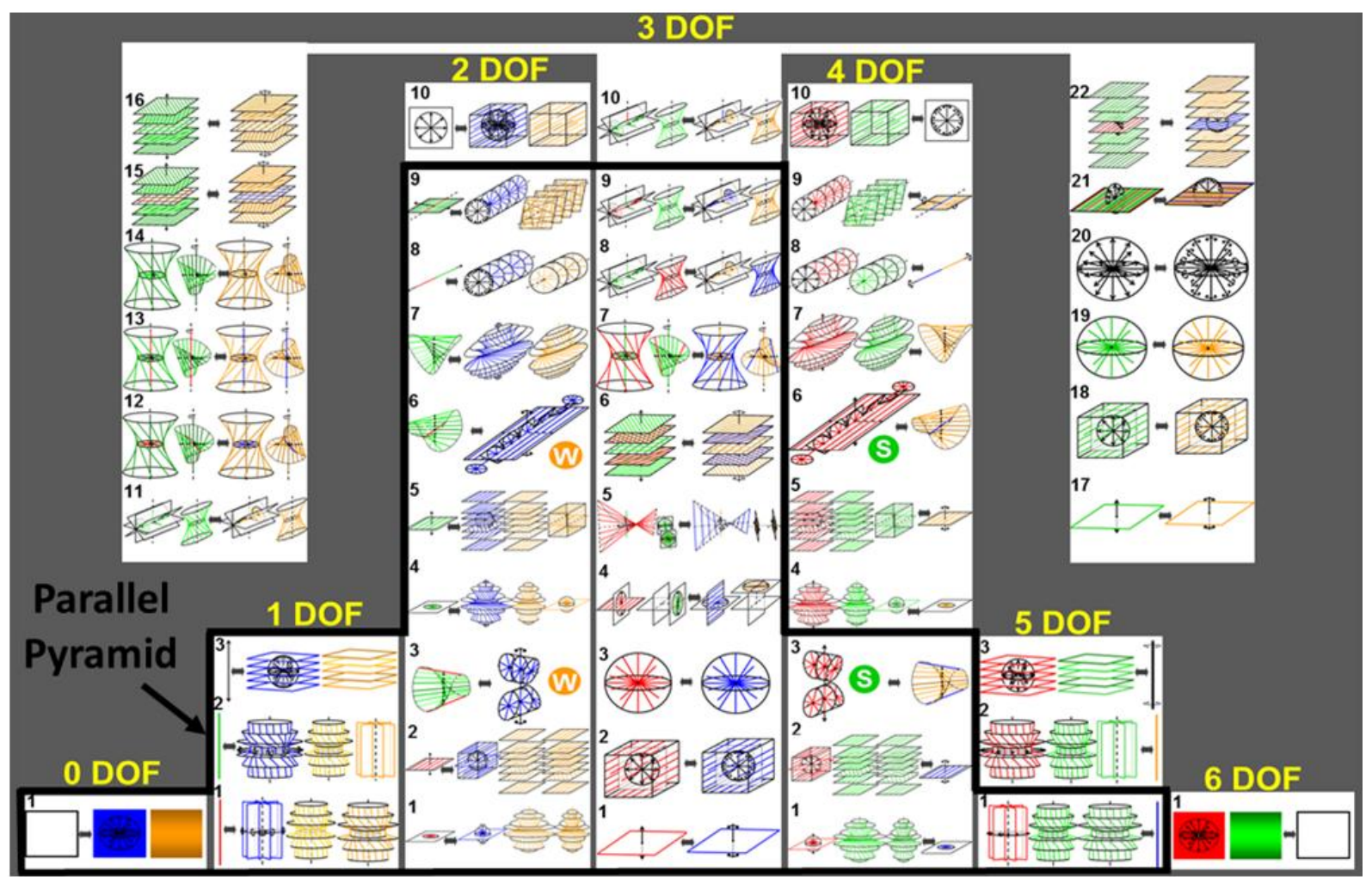

Figure 5. Comprehensive library of freedom and constraint spaces.

the left side of Fig. 4a. This plane of rotation lines and the orthogonal translation arrow is the element's freedom space. Freedom space is a geometric shape that represents all the ways a body may move. It consists of all the twists that result from linearly combining the body's DOF twists.

Every freedom space uniquely links to a complementary or reciprocal space called a constraint space according to the principle of duality (Murray et al., 1994). Constraint space is a geometric shape that represents all the ways a rigid body is constrained by various constraint actions. These actions may be modelled using wrench vectors (Ball, 1900; Hao and McCarthy, 1998). In this paper, pure moment actions are depicted as black lines with circular arrows about their axes, pure force actions are depicted as blue lines called constraint lines (Fig. 2), and coupled moment and force actions are depicted as orange lines. The constraint space of the blade flexure in Fig. 4a is a plane of blue constraint lines and an orthogonal pure moment. This means that the blade can only impose constraining forces along the plane of the blade and a moment perpendicular to this plane. Note that every line that directly joins the blade's rigid bodies and passes entirely through its geometry lies within the blue portion of this constraint space (i.e., the portion that consists of constraint lines).

If a freedom space contains $n$ DOFs (i.e., independent twist vectors), its complementary constraint space will con- tain $m$ independent wrench vectors according to

$6-n=m$.

Note that the constraint space of the blade flexure in Fig. 4a contains three independent wrench vectors (e.g., the pure force wrench vectors $\boldsymbol{W}_{1}, \boldsymbol{W}_{2}$, and $\boldsymbol{W}_{3}$ ). The number of independent wrench vectors within a flexure element's constraint space is called the element's order of constraint. Thus a blade flexure's order of constraint is three.

As another example, consider the wire flexure in Fig. $4 \mathrm{~b}$. Its constraint space is a single constraint line (i.e., pure force wrench vector, $\boldsymbol{W}_{1}$ ) because the wire's geometry is only capable of imposing a single constraining force along its axis. The wire flexure's complementary freedom space consists of (i) a disk of translation arrows that are perpendicular to the axis of the wire, (ii) rotation lines that lie on planes that intersect this axis, and (iii) screw lines that lie on the surfaces of circular hyperboloids and disks (Hopkins, 2010), which are not shown in Fig. $4 \mathrm{~b}$ to avoid visual clutter. Consistent with Eq. (1), the wire flexure's freedom space contains five independent twist vectors or DOFs (e.g., $\boldsymbol{T}_{1}$ through $\boldsymbol{T}_{5}$ shown in Fig. 4b). Note that a wire flexure's order of constraint is one.

There are a finite number of complementary freedom and constraint space pairs called types. These types are provided in Fig. 5 and are described in detail in Hopkins (2010). The specific geometries of these types are not important to understand for the purposes of this paper. What is important to 
recognize is that there are fifty types that are numbered and organized into seven columns that pertain to the number of DOFs contained within each type's freedom space. Note that the freedom and constraint space pair of the blade flexure in Fig. $4 \mathrm{a}$ is Type 1 in the 3 DOF column of Fig. 5 and that the freedom and constraint space pair of the wire flexure in Fig. $4 \mathrm{~b}$ is Type 1 in the 5 DOF column.

The types that lie within the outlined region labelled "Parallel Pyramid" in Fig. 5 contain all of the pertinent freedom and constraint space pairs necessary for analysing and synthesizing parallel flexure systems and elements. In other words, no parallel flexure system or element can achieve the DOFs of the freedom spaces that lie outside of this pyramid. Thus, the freedom and constraint space pairs that correspond with the six parallel flexure elements shown in Fig. 2 all lay within this pyramid. The constraint lines within element $E_{3}$ from Fig. 2 lie within the constraint space of Type 1 in the 1 DOF column of Fig. 5. The constraint lines within element $E_{4}$ from Fig. 2 lie within the constraint space of Type 3 in the 3 DOF column of Fig. 5. The constraint lines within element $E_{5}$ from Fig. 2 lie within the constraint space of Type 7 in the 3 DOF column of Fig. 5. The constraint lines within element $E_{6}$ from Fig. 2 lie within the constraint space of Type 8 in the 2 DOF column of Fig. 5.

Others have classified screw systems similar to those in Fig. 5 for different applications using different criteria. Gibson and Hunt (1990) introduced an approach for classifying screw systems based on projective geometry. Rico and Duffy (1992) proposed a classification based upon the theory of orthogonal spaces and subspaces by examining the characteristics of the reciprocal basis of screw systems. The geometric shapes in Fig. 5 are complete and have been classified to facilitate the rapid analysis and synthesis of parallel, serial, and hybrid flexure systems and elements. Thus, no flexure system or element exists, which cannot be analysed or synthesized using the spaces within this library.

\subsection{Analysing parallel flexure systems}

The following two principles are important for determining the kinematics of any parallel flexure system:

1. The wrench vectors within the constraint spaces of a parallel flexure system's flexure elements may be linearly combined to generate the effective constraint space of the entire system.

2. The effective freedom space of a parallel flexure system consists of the intersection of the freedom spaces of every flexure element within the system. In other words, a parallel flexure system's freedom space consists of the twist vectors that are common among the freedom spaces of the system's flexure elements.

Consider the parallel flexure system that consists of two wire flexures in Fig. 6. According to the first principle, the sys-

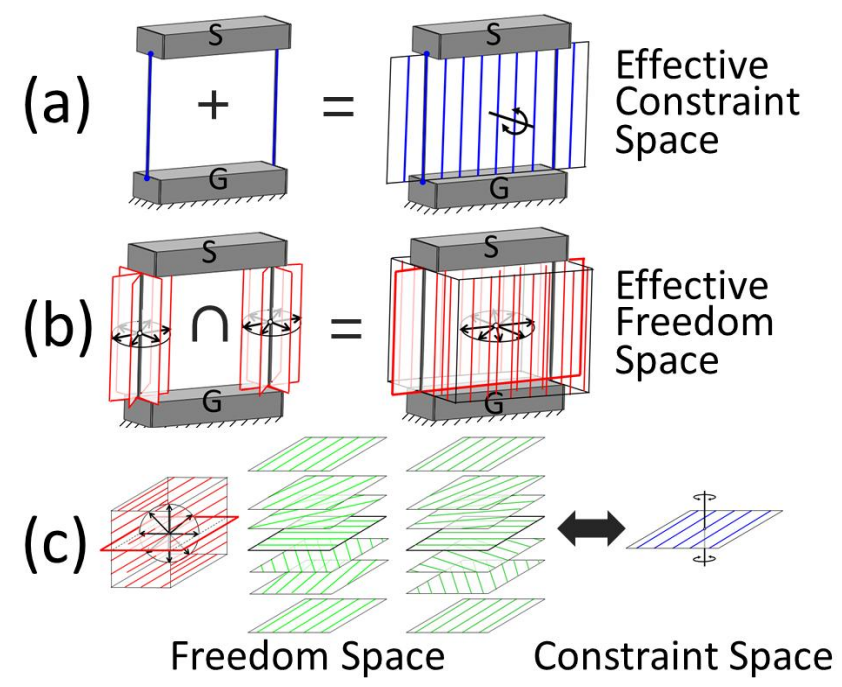

Figure 6. Linearly combine constraints in parallel (a) or find the intersection of their freedom spaces (b) to find the system's complementary freedom and constraint spaces $(\mathbf{c})$.

tem's effective constraint space may be determined by linearly combining the two pure force wrench vectors shown as parallel constraint lines in Fig. 6a. The resulting space is a plane of parallel constraint lines and a pure moment that is perpendicular to this plane as shown on the right side of the figure. According to the second principle, the system's effective freedom space may be determined by identifying the intersection of the freedom spaces of both wire flexures (Fig. 4b) shown in Fig. 6b. The rotation lines that are common among these freedom spaces lie on the plane that contains the two wire flexures and are parallel with the axes of these flexures and thus lie within an infinitely large box as shown. The translation arrows that are common among these freedom spaces lie within a disk that is perpendicular to the axes of the wire flexures. Note that the locations of these translation arrows are unimportant as translation twists are solely directional. The screw lines that are common among these freedom spaces are not shown in Fig. $6 \mathrm{~b}$ to avoid visual clutter. These screw lines, described in Hopkins (2010), are shown with the other translation arrows and rotation lines in the freedom space of Fig. 6c. Note from Fig. 6c that the effective constraint space of the system (Fig. 6a) and its effective freedom space (Fig. 6b) are complementary spaces and are labelled Type 2 in the 4 DOF column of Fig. 5. Moreover, note that it does not matter that the system's effective spaces shown in Fig. 6a and b are oriented differently than they are in Fig. 6c.

\subsection{Synthesizing parallel flexure systems}

There are four steps to synthesize parallel flexure systems: Step 1: identify the system's desired DOFs. 
Step 2: identify the corresponding freedom space from Fig. 5 that contains the DOFs of step 1. This freedom space will result from the linear combination of the twists of those DOFs and is the system's effective freedom space. If this freedom space lies outside of the Parallel Pyramid from the library of Fig. 5, it is not possible to synthesize a parallel flexure system that achieves the desired DOFs from step 1. In this case, the designer must synthesize either a serial or hybrid flexure system to achieve these DOFs.

Step 3: select constraint spaces that lie within the complementary constraint space of the freedom space of step 2 (i.e., the system's effective constraint space). The number of constraint spaces selected will determine the number of parallel flexure elements within the system. These constraint spaces must lie within the Parallel Pyramid of Fig. 5 to the right of the column that contains the system's constraint space. Designers can also select the system's effective constraint space itself as one of these spaces. Any number of viable constraint spaces may be selected as long as the total number of independent wrench vectors from all of the selected spaces combined equals the number of independent wrench vectors within the system's constraint space. The same constraint space may be selected multiple times.

Step 4: generate parallel flexure elements with geometries that lie within the blue portions of these selected constraint spaces (i.e., the regions of the spaces that consist of constraint lines). A subset of constraint lines within each of these spaces should entirely fill and directly pass through the geometry of the space's element and join the system's rigid stage to its fixed ground. This subset of constraint lines should contain as many independent pure force wrench vectors as independent wrench vectors within the entire constraint space from which the element is generated.

If the sum of the order of constraint of every parallel flexure element within the final system equals the number of independent wrench vectors within the system's constraint space, the system is exactly-constrained (Blanding, 1999). If this sum is greater than the number of independent wrench vectors within the system's constraint space, the system is over-constrained.

An example is provided to demonstrate the approach of this section. Suppose for step 1 we wish to synthesize a parallel flexure system that possesses a single rotation DOF as shown in Fig. 7a. For step 2, the freedom space of a rotation DOF is a single rotation line as shown in Fig. 7b. This space is the Type 1 freedom space from the 1 DOF column of Fig. 5. Its complementary constraint space shown in Fig. 7b consists of (i) constraint lines that lie on planes that intersect the rotation line, (ii) a disk of pure moment lines that point in directions that are perpendicular to the axis of the rotation line, and (iii) coupled moment and force lines that lie on the surfaces of circular hyperboloids and disks as described in Hopkins (2010). For step 3, we select two constraint spaces that lie within this constraint space. The two selected spaces are the Type 1 constraint space from the 3



Figure 7. Desired DOFs (a) belong to a freedom space that links to a complementary constraint space (b) within which flexure elements may be selected (c).

DOF column of Fig. 5 shown in Fig. 4a. We orient these two planar constraint spaces within the system's effective constraint space as shown in Fig. 7c. For step 4, we use the region of these spaces that consists entirely of constraint lines to generate flexure blades that connect the system's stage to its fixed ground as shown. The resulting parallel flexure system (Fig. 7c) achieves the desired rotation DOF. Note, however, that the system is over-constrained because its effective constraint space (Fig. 7b) consists of five independent wrench vectors while the sum of the order of constraint of each parallel flexure element within the system is six. Recall that the order of constraint of a flexure blade is three.

\subsection{Analysing serial flexure systems}

The following two principles are important for determining the kinematics of any serial flexure system:

1. The twist vectors within the freedom spaces of the parallel flexure system constituents may be linearly combined to generate the effective freedom space of the entire serial flexure system.

2. The effective constraint space of a serial flexure system consists of the intersection of the constraint spaces of the parallel flexure system constituents within the system. In other words, a serial flexure system's constraint space consists of the wrench vectors that are common among the constraint spaces of the parallel flexure system constituents.

Consider the serial flexure system in Fig. 8 that consists of the two parallel flexure systems from Figs. 6 and 7c stacked together. According to the first principle, the system's effective freedom space may be determined by linearly combining 
(a)

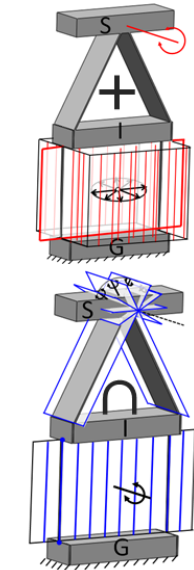

(c)

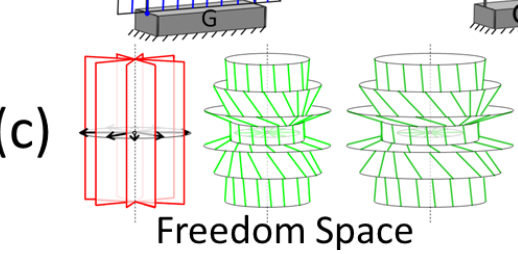

Freedom Space

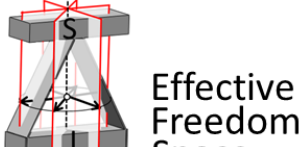

Space

(b)

Figure 8. Linearly combine freedom spaces in series (a) or find the intersection of their constraint spaces (b) to find the system's complementary freedom and constraint spaces (c).

the twist vectors within the two freedom spaces of Figs. $6 \mathrm{~b}$ and 7a as shown in Fig. 8a. The resulting space (Fig. 8a) is the same freedom space shown in Fig. 4b. According to the second principle, the system's effective constraint space may be determined by identifying the intersection of the constraint spaces of both parallel flexure system constituents shown in Figs. 6a and 7c and again in Fig. 8b. The only wrench vector that is common among these constraint spaces is the constraint line shown in Fig. 8b. Note from Fig. 8c that the effective freedom space of the serial flexure system (Fig. 8a) and its effective constraint space (Fig. 8b) are complementary spaces and are labelled Type 1 in the 5 DOF column of Fig. 5. Moreover, note that the circular hyperboloids and disks that contain the screw lines of the freedom space mentioned previously are also shown in Fig. 8c.

\subsection{Synthesizing serial flexure systems}

There are five steps to synthesize serial flexure systems:

Step 1: identify the system's desired DOFs.

Step 2: identify the corresponding freedom space from Fig. 5 that contains the DOFs of step 1. This freedom space will result from the linear combination of the twists of those DOFs and is the system's effective freedom space.

Step 3: select intermediate freedom spaces from within the freedom space of step 2 . These intermediate freedom spaces correspond with the freedom spaces of the parallel flexure system constituents within the serial system being synthesized. Thus, the number of intermediate freedom spaces selected will determine the number of parallel flexure system constituents within the overall system. These freedom spaces must lie within the Parallel Pyramid of Fig. 5 to the left of the column that contains the system's freedom space. Designers can also select the system's effective freedom space itself as one of these spaces as long as it lies within the Parallel Pyramid. Any number of viable intermediate freedom spaces may be selected as long as the total number of independent twist vectors from all of the selected spaces combined equals the number of independent twist vectors within the system's freedom space. The same freedom space may be selected multiple times.

Step 4: select constraint spaces that lie within the complementary constraint spaces of the selected intermediate freedom spaces from step 3 (i.e., the system's intermediate constraint spaces). The number of constraint spaces selected from each intermediate constraint space will determine the number of parallel flexure elements within each parallel flexure system constituent. These constraint spaces must lie within the Parallel Pyramid of Fig. 5 to the right of the column that contains the intermediate constraint space from which they are selected. Designers can also select the intermediate constraint space itself as one of these spaces. Any number of viable constraint spaces may be selected as long as the total number of independent wrench vectors from all of the selected spaces combined equals the number of independent wrench vectors within the intermediate constraint space from which they are selected. The same constraint space may be selected multiple times.

Step 5: generate parallel flexure elements with geometries that lie within the selected constraint spaces from step 4 . These geometries must be selected from within the blue regions of these spaces (i.e., the regions that consist of constraint lines only). A subset of constraint lines within each of these spaces should entirely fill and directly pass through the geometry of the space's element and join the appropriate rigid bodies within the system. This subset of constraint lines should contain as many independent pure force wrench vectors as independent wrench vectors within the entire intermediate constraint space from which the element is generated. The rigid bodies that a particular element should join pertain to the intermediate constraint space from which the element's constraint space was selected. Ultimately, the stage of the serial flexure system should be joined from one intermediate rigid body to the next by the elements from each intermediate constraint space until the system is joined to a fixed ground.

If the sum of the order of constraint of every parallel flexure element within one or more parallel flexure system constituents within the serial system is greater than the number of independent wrench vectors within the constraint space of the corresponding parallel flexure system constituent, the overall serial system is over-constrained.

If, however, the sum of the number of DOFs within each selected intermediate freedom space from step 3 exceeds the number of total independent twist vectors within all of these 


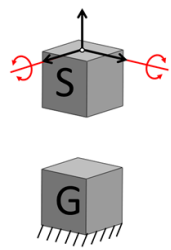

(a)

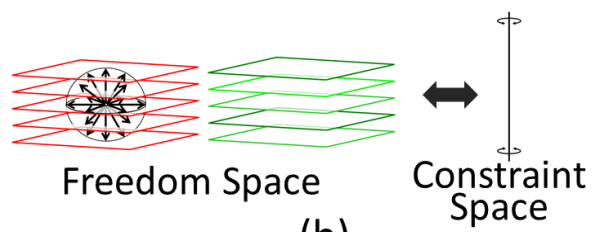

(b)

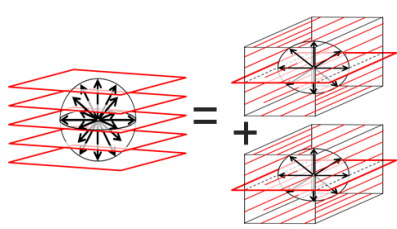

(c)

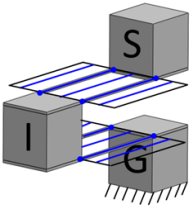

(d)

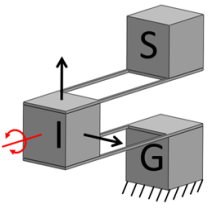

(e)
Figure 9. Desired DOFs (a), system's freedom and constraint space (b), intermediate freedom spaces selected from the system's freedom space (c), flexure elements selected from the intermediate constraint spaces (d), and the intermediate body's underconstrained DOFs (e).

spaces combined, the system is under-constrained (Blanding, 1999; Hopkins and Culpepper, 2011). The intermediate rigid bodies of an under-constrained system often possess DOFs that are not constrained even when the final system's stage is held fixed relative to its ground. As such, the performance of an under-constrained system suffers from unwanted vibrations and poor controllability. Note that only serial and hybrid flexure systems are susceptible to under-constraint because, unlike parallel flexure systems, serial and hybrid systems possess intermediate rigid bodies.

An example is provided to demonstrate the approach of this section. Suppose for step 1 we wish to synthesize a serial flexure system that possesses five DOFs - three translations and two rotations as shown in Fig. 9a. For step 2, the freedom space of these five DOFs consists of (i) rotation lines that lie on parallel planes, (ii) translation arrows that point in all directions as shown by the sphere in Fig. 9b, and (iii) screw lines that lie on the same parallel planes as the rotation lines. This freedom space is the Type 3 space from the 5 DOF column of Fig. 5. Note that its complementary constraint space is a pure moment that is perpendicular to the parallel planes of the freedom space and points along the axis of the rotation that we wish to constrain. For step 3, we select two intermediate freedom spaces that lie within the system's freedom space and orient them as shown in Fig. 9c with one on top of the other. Each of these selected spaces is the Type 2 freedom space from the 4 DOF column of Fig. 5 shown in Fig. 6c. For step 4, we note that the complementary constraint space of each these intermediate freedom spaces is the planar constraint space of parallel constraint lines described in Fig. 6a and c. We select two constraint spaces from within each of these intermediate constraint spaces of parallel constraint lines (Fig. 9d). Each of these constraint spaces is a single constraint line and is the Type 1 constraint space from the 5 DOF column of Fig. 5 shown in Fig. 8c. For step 5 , we use these constraint lines to generate wire flexures. The two wire flexures generated from the intermediate constraint space on the top join the system's stage to an intermediate rigid body. The two wire flexures generated from the intermediate constraint space on the bottom join the same intermediate rigid body to a fixed ground as shown in Fig. 9d. The final serial flexure system shown in Fig. 9e achieves the desired five DOFs. Note that the system is not over-constrained because the sum of the order of constraint of both wire flexures in each intermediate constraint space is two and two is the number of independent wrench vectors within each of these spaces. The system is, however, under-constrained because the sum of the DOFs from both intermediate freedom spaces selected in Fig. $9 c$ is eight (i.e., four from each) but the total number of independent twist vectors within these two spaces combined is only five. Note, therefore, from Fig. 9e that when the system's stage is held fixed with respect to its ground, the intermediate rigid body possesses three DOFs that are not constrained - two translations and one rotation. Although this system is under-constrained and not practical to use in its current form, we will return to it in a later section to make it more practical as a hybrid flexure element.

\section{Hybrid flexure systems and elements}

This section applies the principles of the previous section to enable the analysis and synthesis of hybrid flexure systems and elements.

\subsection{Analysing hybrid flexure systems}

The stage of a general hybrid flexure system is joined to ground via flexure limbs arranged in parallel. Each limb can be a parallel, serial, or hybrid flexure system or element. As the limbs of a hybrid system are arranged in parallel, the principles for analysing their kinematics are similar to those given in Sect. 2.2 for analysing parallel flexure systems. That is, the wrench vectors within the effective constraint spaces of each limb may be linearly combined to generate the constraint space of the entire hybrid system, and the hybrid system's freedom space is the intersection of each limb's effective freedom space.

As an example, consider the hybrid flexure system in Fig. 10 that consists of two limbs arranged in parallel. Each limb is the serial flexure system from Fig. 8. The hybrid system's effective constraint space may be determined by linearly combining the two pure force wrench vectors from the effective constraint spaces of each limb (Fig. 8b) as shown in Fig. 10a. The resulting space is the same as that shown in Fig. 6a. The system's effective freedom space may be determined by identifying the intersection of the effective freedom spaces of both serial flexure systems (Fig. 8a) as shown in Fig. 10b. The resulting space is the same as that shown in 


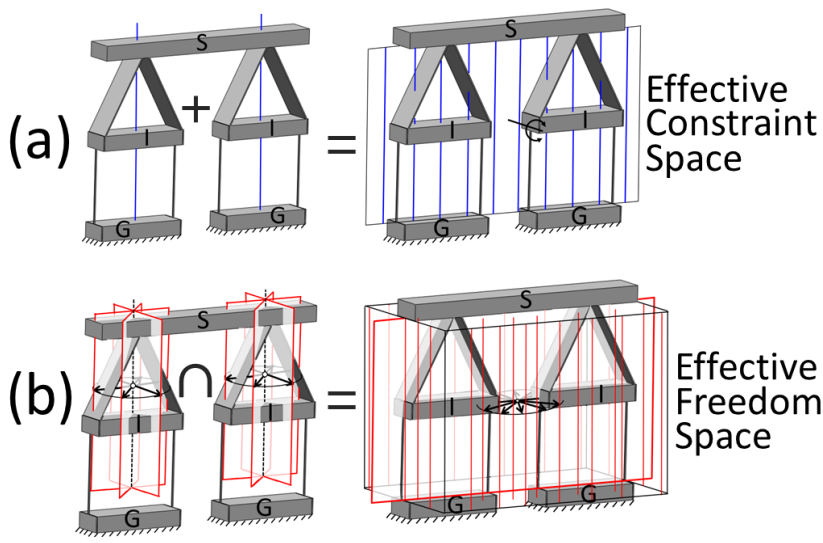

Figure 10. Linearly combine the constraint spaces of limbs (a) or find the intersection of their freedom spaces (b) to find a hybrid system's complementary freedom and constraint spaces.

Fig. 6b. The hybrid system's complementary spaces are thus the Type 2 spaces in the 4 DOF column of Fig. 5 shown in Fig. 6c.

\subsection{Synthesizing hybrid flexure systems}

There are five steps to synthesize general hybrid flexure systems:

Step 1: identify the system's desired DOFs.

Step 2: identify the corresponding freedom space from Fig. 5 that contains the DOFs of step 1. This freedom space will result from the linear combination of the twists of those DOFs and is the system's effective freedom space.

Step 3: select constraint spaces that lie within the complementary constraint space of the freedom space from step 2 (i.e., the system's constraint space). Each limb of the hybrid flexure system is generated from one of these constraint spaces. These constraint spaces can belong to any column within the library of Fig. 5 to the right of the column that contains the system's constraint space. Note that these spaces do not need to lie within the Parallel Pyramid of Fig. 5 unless they are intended to be used to generate a parallel limb (i.e., a limb that consists of parallel flexure elements that directly connect the system's stage to ground). Designers can also select the system's constraint space itself as one of these spaces. Any number of viable constraint spaces may be selected as long as the total number of independent wrench vectors from all of the selected spaces combined equals the number of independent wrench vectors within the system's constraint space. The same constraint space may be selected multiple times.

Step 4: determine what type of limb each constraint space selected from step 3 is intended to generate. If the constraint space is intended to generate a parallel limb and it belongs within the Parallel Pyramid of Fig. 5, the designer should determine this space's complementary freedom space and use that space to continue on with steps 3 through 4 in Sect. 2.3 to generate the limb. If the constraint space is intended to generate a serial limb, the designer should determine this space's complementary freedom space and use that space to continue on with steps 3 through 5 in Sect. 2.5 to generate the limb. If the constraint space is intended to generate a hybrid limb, the designer should first create a serial limb using the instructions from the previous sentence and then continue on to the next step to make the limb hybrid. If none of the limbs are intended to be hybrid, the final system is complete with this step.

Step 5: identify a parallel flexure element or a group of parallel flexure elements within a parallel flexure system constituent within the desired serial limb from step 4 and linearly combine the wrench vectors within the constraint spaces of these elements to generate an effective constraint space for that group of elements. Then identify this space's complementary freedom space using the library of Fig. 5 and apply this freedom space to steps 3 through 5 in Sect. 2.5 to generate a serial flexure system within the parallel flexure system constituent of the original serial limb. Designers can use this step multiple times with different groups of elements within the same parallel flexure system constituent or within different parallel flexure system constituents within the same serial limb to change it into a hybrid limb of any variety.

If the sum of the number of independent wrench vectors within each individual constraint space selected from step 3 is greater than the number of independent wrench vectors within the hybrid system's overall constraint space, or if the sum of the order of constraint of every parallel flexure element within one or more parallel flexure system constituents within the hybrid system is greater than the number of independent wrench vectors within the constraint space of the corresponding parallel flexure system constituent, the overall hybrid system is over-constrained.

If, however, any limbs within a hybrid flexure system contain one or more serial flexure systems that are underconstrained according to the criteria of Sect. 2.5, the entire hybrid system is also underconstrained as it will contain intermediate bodies that are not constrained when its final stage is held fixed with respect to its ground.

An example is provided to demonstrate the approach of this section. Suppose for step 1 we wish to synthesize a hybrid flexure system that possesses a single translation DOF. For step 2, the freedom space of this DOF is a translation arrow shown in Fig. 11a. This freedom space is the Type 3 space from the 1 DOF column of Fig. 5. Note that its complementary constraint space consists of (i) constraint lines that lie on parallel planes, (ii) pure moments that point in all directions as shown by the sphere in Fig. 11a, and (iii) coupled moment and force lines that lie on the same parallel planes as the constraint lines. For step 3, we select two constraint spaces that lie within the system's constraint space oriented as shown in Fig. 11b. Each of these spaces is the Type 2 constraint space from the 2 DOF column of Fig. 5 shown in 
(a)

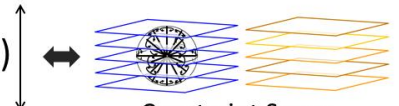

Freedom
Space

Constraint Space

(c)

Freedom

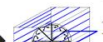

Space

Constraint Space

(e)



(c) (b)

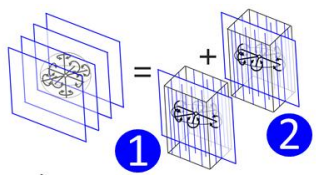

(d)

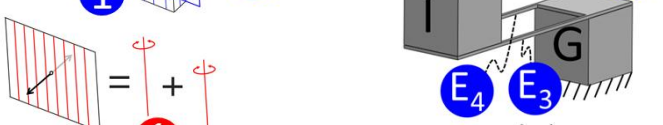

(a)
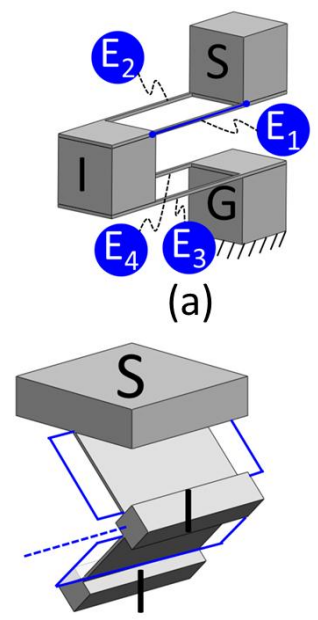

(h)

(f)

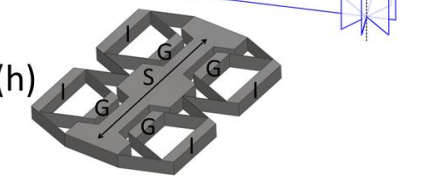

Figure 11. Desired system freedom and constraint spaces (a), selecting the limbs' constraint spaces (b), complementary spaces (c), selecting intermediate freedom spaces (d), synthesizing flexure elements from intermediate constraint spaces (e) and (f), synthesized hybrid system (g), and symmetric version (h).

Fig. 11c. This constraint space consist of (i) a plane of constraint lines, (ii) a box of parallel constraint lines, (iii) a disk of pure moments that are perpendicular to these parallel constraint lines, and (iv) coupled moment and force lines that lie on parallel planes as described in Hopkins (2010). The complementary freedom space of this constraint space consists of a plane of parallel rotation lines and a perpendicular translation arrow as shown in Fig. 11c. For step 4, we decide to generate serial limbs from the selected constraint spaces labelled 1 and 2 in Fig. 11b. Thus, according to the instructions given in step 4 , we continue on with steps 3 through 5 in Sect. 2.5 using the complementary freedom space in Fig. 11c to generate the serial limbs. We first generate the limb that corresponds with the constraint space labelled 1 in Fig. $11 \mathrm{~b}$. For step 3 in Sect. 2.5, we select two intermediate freedom spaces that lie within the freedom space of Fig. 11c and orient them as shown in Fig. 11d. Each of these spaces is the Type 1 freedom space from the 1 DOF column of Fig. 5 shown in Fig. 7b. For step 4 in Sect. 2.5, we note that the complementary constraint spaces of these intermediate freedom spaces are also shown in Fig. 7b. We first focus on the complementary constraint space of the intermediate freedom space labelled 1 in Fig. 11d. We select two constraint spaces from within this space as shown in Fig. 11e. Both constraint spaces consist of a plane of constraint lines and a perpendicular moment. Each of these spaces is the Type 1 constraint space from the 3 DOF column of Fig. 5 shown in Fig. 4a. For step 5 in Sect. 2.5, we use the blue portion of these spaces (i.e., the region that consists of constraint lines) to generate two blade flexures shown in Fig. 11e. These flexures join a grounded body to an intermediate body. We return to the complementary constraint space of the intermediate freedom space la-

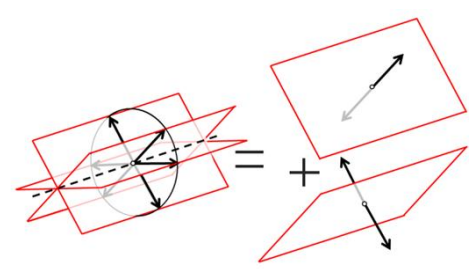

(b)

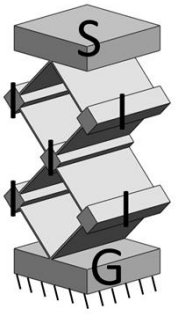

(d)

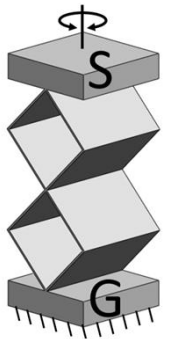

(e)
Figure 12. Element $E_{1}$ selected to make serial (a), intermediate freedom spaces selected within $E_{1}$ 's effective freedom space (b), generating blade flexure elements within the intermediate constraint spaces (c), equivalent hybrid flexure system (d), equivalent hybrid flexure element (e).

belled 2 in Fig. 11d and repeat the same process to generate the two flexure blades shown in Fig. 11f. These blade flexures join the intermediate body to the system's stage. We now return to the constraint space labelled 2 in Fig. $11 \mathrm{~b}$ to generate the system's other serial limb. We follow the same steps used to generate the limb of Fig. 11f to synthesize a similar limb shown in Fig. 11g. A more symmetric version of the system is shown in Fig. 11h (Awtar et al., 2007). Note that the system is over-constrained but not under-constrained. Thus, if the system's stage is held fixed with respect to its four grounded bodies, all of its intermediate rigid bodies will be fully constrained.

As the hybrid system in Fig. 11h consists only of serial limbs, step 5 from this section was not necessary to perform. Suppose, however, we wish to change the serial flexure system in Fig. 9e into a hybrid system. This process would require step 5 and is thus a good example to demonstrate how to change a serial limb into a hybrid limb. According to step 5 , therefore, we select the wire flexure element $E_{1}$ shown in Fig. 12a as the element to change. This element's effective constraint space is the single constraint line shown in the figure. Its complementary freedom space is the Type 1 space from the 5 DOF column of Fig. 5 and is shown in Fig. 8c. Using this freedom space, we continue on with steps 3 through 5 in Sect. 2.5. For step 3 in Sect. 2.5, we select two intermediate freedom spaces that lie within this space and orient them as shown in Fig. 12b. Each of these spaces is the Type 1 freedom space from the 3 DOF column of Fig. 5 shown in Fig. 4a. For step 4 in Sect. 2.5, we note that the complementary constraint spaces of these intermediate freedom spaces are planes of constraint lines with orthogonal pure moments 
(Fig. 4a). We select these spaces to generate the elements for the next step. For step 5 in Sect. 2.5, we use the region of these spaces that consists of constraint lines only to generate the two stacked blade flexures shown in Fig. 12c. These stacked blade flexures constitute a serial flexure system that possesses the same effective freedom and constraint space as the wire flexure element $E_{1}$ from Fig. 12a. Thus, the serial flexure system of Fig. 12c may replace element $E_{1}$ from Fig. 12a as long as the dashed line at the intersection of the blades shown in Fig. 12c is collinear with the axis of the wire. We could thus repeat step 5 from this section for the remaining three elements shown in Fig. 12a to change all the elements into the same four serial flexure systems from Fig. 12c as shown in Fig. 12d.

The resulting system is a hybrid system that achieves the same five DOFs (Fig. 9a) as the serial system of Fig. 12a. This hybrid system possesses more symmetry, is easier to fabricate, and is more effective in constraining the unwanted axial rotation than the system from Fig. 12a. Unfortunately, however, the hybrid system of Fig. 12d is under-constrained like the system in Fig. 12a. Thus if it were used as a flexure coupling, for instance, to pass only the torque of one spinning shaft through to the next, the hybrid system's intermediate rigid bodies would vibrate with multiple unwanted DOFs. This issue could be mitigated by effectively eliminating the intermediate rigid bodies within the system as shown in Fig. 12e. This reduction in mass significantly improves the flexure's dynamic performance. Note also that without intermediate rigid bodies, the flexure is no longer considered a system but an element because its entire geometry is compliant and acts as a single constraining unit. The element of Fig. 12e is thus a hybrid flexure element.

The process for generating serial and hybrid flexure elements is twofold. First, designers must use the approach of this paper to synthesize a serial or hybrid flexure system that possesses the element's desired DOFs. Second, designers must eliminate the system's intermediate rigid bodies to produce the desired element. It is important to recognize, however, that not all serial or hybrid systems possess intermediate rigid bodies that can be eliminated like the system of Fig. 12d. The intermediate rigid body of the serial system from Fig. 12a, for instance, could not be eliminated without changing the system's desired kinematics. Designers should thus synthesize systems with intermediate rigid bodies that can be eliminated.

Ten hybrid flexure element examples are provided in Fig. 13. These elements are each shown with their effective constraint space. Both elements $E_{1}$ and $E_{2}$ are the Type 3 constraint space from the 5 DOF column of Fig. 5. Note, therefore, that element $E_{2}$ can also be used as a flexure coupling (Soemers, 2010). Element $E_{3}$ is the Type 1 space from the 5 DOF column of Fig. $5, E_{4}$ is the Type 1 space from the $1 \mathrm{DOF}$ column, $E_{5}$ is the Type 1 space from the $6 \mathrm{DOF}$ column, both $E_{6}$ and $E_{7}$ are the Type 1 space from the $3 \mathrm{DOF}$

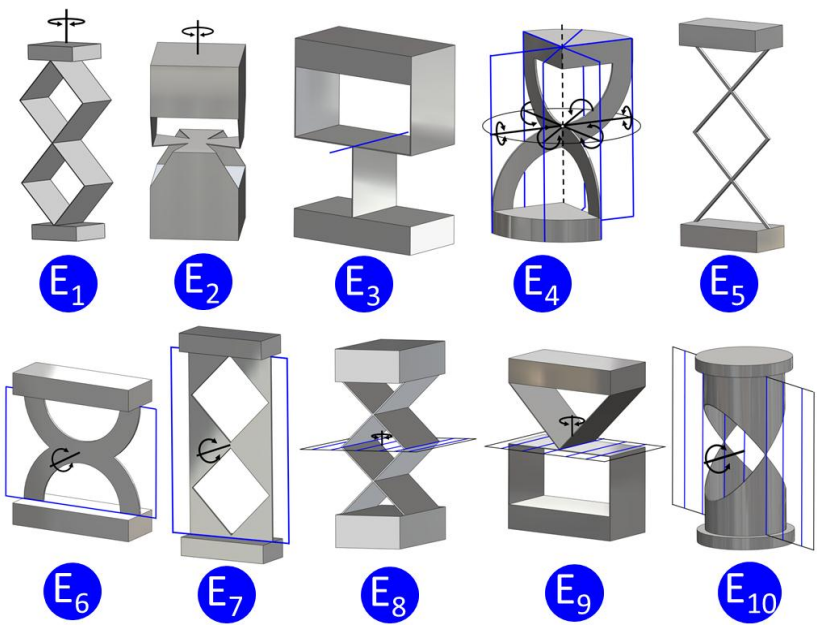

Figure 13. Hybrid flexure element examples.

column, and $E_{8}, E_{9}$ and $E_{10}$ are the Type 2 space from the 4 DOF column.

Having access to a large variety of flexure elements like those provided in Fig. 13 can help facilitate the rapid synthesis of hybrid flexure systems. Suppose, for instance, we wished to synthesize a hybrid flexure system that possesses three translations as shown in Fig. 14a. The system's freedom space is thus the sphere of translation arrows shown in Fig. 14b. This space is the Type 20 space from the 3 DOF column of Fig. 5. Its complementary constraint space is the sphere of pure moments also shown in Fig. 14b. If we desired the system to possess three limbs we could select the three constraint spaces shown in Fig. 14c. These spaces are each pure moments that point in different directions. As the effective constraint space of the hybrid flexure element from Fig. 12e is a single pure moment that points along the element's axis, we could align the axes of three of these elements with the axes of the three pure moments selected in Fig. 14c as shown in Fig. 14d. The resulting flexure system achieves the three desired translations over relatively large ranges with minimal parasitic error. It is symmetric and easy to fabricate using conventional processes (e.g., waterjet, wire EDM, and milling) and its topology allows for symmetric force-based actuation as shown by the blue arrows in Fig. 14e. Note that although the system of Fig. 14d consists of a single rigid stage joined directly to a fixed ground by flexure elements, the system is not considered parallel but hybrid. The reason for this classification is that the elements that join the stage to the ground are hybrid elements.

As a final note, it is important to emphasize that the theory of this paper enables designers to analyse and synthesize general hybrid flexure systems that consist of combinations of parallel and serial flexure systems and elements only. It does not enable designers to analyse and synthesize hybrid flexure systems with interconnected limbs (i.e., the intermediate rigid bodies within one or more limbs are joined to the 


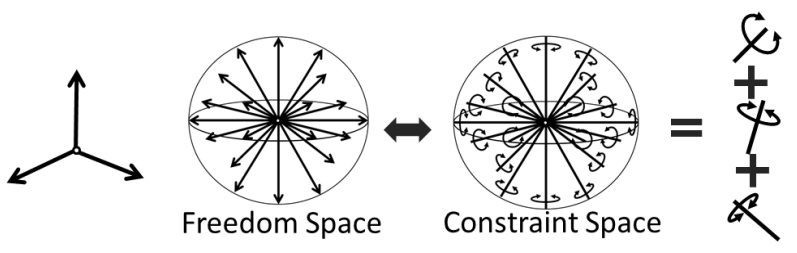

(a)

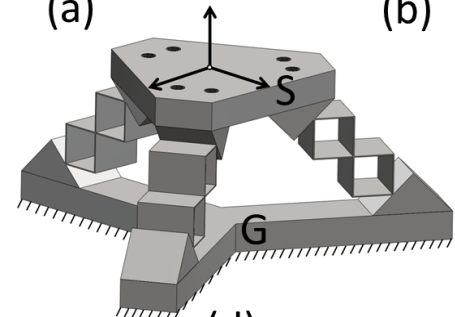

(d) (b)

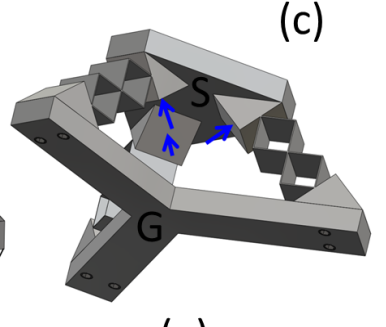

(e)

Figure 14. Desired DOFs (a), the system's freedom and constraint space (b), three limb constraint spaces (c), final hybrid system (d), and locations for actuating the system (e).

intermediate rigid bodies of other limbs by various flexure systems or elements). The principles for analysing and synthesizing this type of hybrid system are the topic of a future paper.

\section{Conclusions}

In this paper, we extend the principles of the FACT design approach such that designers can analyse and synthesize hybrid flexure systems and elements using geometric shapes. These shapes enable designers to rapidly generate and compare a large variety of non-intuitive flexure solutions that achieve any desired set of DOFs. Guidelines are provided for helping designers utilize or avoid over-, under-, and exact-constraint in the systems they synthesize. Hybrid flexure elements are introduced as useful alternatives to the standard parallel flexure elements widely used by flexure designers (e.g., wire, blade, living hinge, and notch flexures). Ten hybrid flexure element examples are provided, and example hybrid flexure systems are synthesized as case studies. One of these examples is a novel system that achieves three translations.

Acknowledgements. This work was performed under the auspices of the US Department of Energy by Lawrence Livermore National Laboratory under Contract DE-AC52-07NA27344. LLNL-JRNL-627952.

Edited by: H. Su

Reviewed by: J. Yu and two anonymous referees

\section{References}

Awtar, S., Slocum, A. H., and Sevincer, E.: Characteristics of beambased flexure modules, J. Mech. Design, 129, 625-639, 2007.

Awtar, S., Ustick, J., and Sen, S.: An XYZ parallel kinematic flexure mechanism with geometrically decoupled degrees of freedom, Journal of Mechanisms and Robotics, 5, 015001, doi:10.1115/1.4007768, 2012.

Ball, R. S.: A Treatise on the Theory of Screws, Cambridge University Press, Cambridge, UK, 1900.

Blanding, D. L.: Exact Constraint: Machine Design Using Kinematic Principles, New York, NY, ASME Press, 1999.

Bothema, R. and Roth, B.: Theoretical Kinematics, Dover, New York, 1990.

Gibson, C. G. and Hunt, K. H.: Geometry of screw systems-II, classification of screw systems, Mech. Mach. Theory, 25, 11-27, 1990.

Hao, G. and Kong, X.: A normalization-based approach to the mobility analysis of spatial compliant multi-beam modules, Mech. Mach. Theory, 59, 1-19, 2013.

Hao, F. and McCarthy, J. M.: Conditions for line-based singularities in spatial platform manipulators, J. Robotic Syst., 15, 43-55, 1998.

Hopkins, J. B.: Design of parallel flexure systems via freedom and constraint topologies (FACT), M.S., Massachusetts Institute of Technology, Cambridge, M.A., 2007.

Hopkins, J. B.: Design of flexure-based motion stages for mechatronic systems via freedom, actuation and constraint topologies (FACT), Ph.D. thesis, Massachusetts Institute of Technology, Cambridge, M.A., 2010.

Hopkins, J. B.: Modelling and generating new flexure constraint elements, Proc. of the 12th International Conference of the European Society for Precision Engineering \& Nanotechnology, Stockholm, Sweden, 4-8 June 2012.

Hopkins, J. B.: Analysing and designing serial flexure elements, accepted to Proc. of the ASME 2013 International Design Engineering Technical Conferences \& Computers and Information in Engineering Conference IDETC/CIE 2013, Portland, O.R., August 2013.

Hopkins, J. B. and Culpepper, M. L.: A quantitative, constraintbased design method for multi-axis flexure stages for precision positioning and equipment, Proc. of the Annual Meeting of the American Society for Precision Engineering, Monterey, C.A., 15-20 October 2006.

Hopkins, J. B. and Culpepper, M. L.: Synthesis of multi-degree of freedom, parallel flexure system concepts via freedom and constraint topology (FACT) - part II: Practice, Precis. Eng., 34, 271278, 2010.

Hopkins, J. B. and Culpepper, M. L.: Synthesis of precision serial flexure systems using freedom and constraint topologies (FACT), Precis. Eng., 35, 638-649, 2011.

Hopkins, J. B. and Panas, R. M.: Design of flexure-based precision transmission mechanisms using screw theory, Precis. Eng., 37, 299-307, 2013.

Hopkins, J. B., Vericella, J. J., and Harvey, C. D.: Modelling and generating parallel flexure elements, Precis. Eng., in review, 2013. 
Howell, L. L., Magleby, S. P., and Olsen, B. M.: Handbook of Compliant Mechanisms, John Wiley \& Sons, San Francisco, C.A., 2013.

Hunt, K. H.: Kinematic Geometry of Mechanisms, Oxford University Press, London, UK, 1978.

Klein, F.: Die Allgemeine Lineare Transformation der Linienkoordinaten, Gesammelte math. Abhandlungen I, Springer, Berlin, Germany, 1921.

Kong, X. and Gosselin, C. M.: Type synthesis of 3-DOF translational parallel manipulators based on screw theory, J. Mech. Des.-T. ASME, 126, 83-92, 2004.

$\mathrm{Li}, \mathrm{Y}$. and $\mathrm{Xu}, \mathrm{Q}$.: Design and analysis of a totally decoupled flexure-based XY parallel micromanipulator, IEEE T. Robot., 25, 645-657, 2009.

Merlet, J. P.: Singular configurations of parallel manipulators and Grassmann geometry, Int. J. Robot. Res., 8, 45-56, 1989.

Merlet, J. P.: Parallel Robots, Kluwer Academic Publishers, Norwell, M.A., 2000.

Murray, R. M., Li, Z., and Sastry, S. S.: A Mathematical Introduction to Robotic Manipulation, CRC Press LLC, Boca Raton, F.L., 1994.

Phillips, J.: Freedom in Machinery: Volume 1, Introducing Screw Theory, Cambridge University Press, New York City, N.Y., 1984.

Phillips, J.: Freedom in Machinery: Volume 2, Screw Theory Exemplified. Cambridge University Press, New York City, N.Y., 1990.
Rico, J. M. and Duffy, J.: Classification of screw systems - II: Three-systems, Mech. Mach. Theory, 27, 471-490, 1992.

Smith, S. T.: Flexures: Elements of elastic mechanisms, Gordon and Breach Science Publishers, Newark, N.J., 2000.

Soemers, H. M. J. R.: Design Principles for Precision Mechanisms, T-point Print, Enschede, 2010.

$\mathrm{Su}, \mathrm{H} .:$ Mobility analysis of flexure mechanisms via screw algebra, Journal of Mechanisms and Robotics, 3, 041010, doi:10.1115/1.4004910, 2011.

$\mathrm{Su}, \mathrm{H}$. and Tari, H.: Realizing orthogonal motions with wire flexures connected in parallel, Journal of Mechanisms and Robotics, 132, 121002, doi:10.1115/1.4002837, 2010.

Su, H., Dorozhkin, D. V., and Vance, J. M.: A screw theory approach for the conceptual design of flexible joints for compliant mechanisms, Journal of Mechanisms and Robotics, 1, 041009, doi:10.1115/1.3211024, 2009.

Yu, J. J., Li, S. Z., Pei, X., Su, H., Hopkins, J. B., and Culpepper, M. L.: Type synthesis principle and practice of flexure systems in the framework of screw theory part I: General methodology, Proc. of the ASME 2010 International Design Engineering Technical Conferences \& Computers and Information in Engineering Conference IDETC/CIE 2010, Montreal, Quebec, Canada, 1518 August 2010. 